\title{
Qualidade microbiológica das plantas medicinais produzidas no Estado do Paraná
}

\author{
Zaroni, M. ; Pontarolo, R.; Abrahão, W.S.M.; Fávero, M.L.D; Correa Júnior, C.; \\ Stremel, D.P. \\ Departamento de Farmácia, Universidade Federal do Paraná, Curitiba, PR.
}

\begin{abstract}
RESUMO: Foram analisadas 72 amostras de plantas medicinais, enviadas por produtores de sete regiões do Estado do Paraná, segundo metodologia preconizada pela Organização Mundial da Saúde (OMS). Os resultados das análises microbiológicas realizadas (contagem de microrganismos aeróbios viáveis, contagem de bolores e leveduras, pesquisa de enterobactérias, Escherichia coli, Salmonella sp., Staphylococcus aureus e Pseudomonas aeruginosa indicaram que a maioria das amostras (79\%) não atendia os parâmetros estabelecidos pela OMS, tanto para utilização da planta medicinal na forma de chá ou para uso tópico quanto para uso interno. A maioria das amostras foi reprovada pelo fato de apresentar contagens de microrganismos aeróbios e de bolores e leveduras elevadas. Tal reprovação evidencia a necessidade de um programa de treinamento dos produtores, envolvendo as diversas etapas de produção e o posterior processamento.
\end{abstract}

Unitermos: qualidade microbiológica; plantas medicinais.

ABSTRACT: Microbiological quality of medicinal plants produced by the State of Paraná (Brazil). 72 samples of medicinal plants produced at seven different regions of Paraná State, Brazil, were analysed according to the World Health Organization methodology. The results of the microbiological analysis (total viable aerobic count, yeasts and moulds count, detection of Enterobacteriaceae and other Gram-negative bacteria: Escherichia coli, Salmonella sp., Pseudomonas aeruginosa and Staphylococcus aureus indicated that the majority of the samples $(79 \%)$ is not in accord to World Health Organization parameters for medicinal plants to be used for herbal tea or topic and internal uses. The main reason of this poor microbiological quality was due to aerobic microrganisms and yeasts and moulds counting. It is an evidence that producers must be orientated by capable professionals in every stage of production in order to provide the necessary quality of the raw material to further medicinal usage.

Key-words: microbiological quality; medicinal plants. 


\section{INTRODUÇÃO}

Os efeitos indesejáveis causados pelo uso abusivo dos medicamentos sintéticos e o elevado custo de tais medicamentos ocasionaram o aumento do consumo de medicamentos de origem vegetal (LUZ et al., 1997/1998; FETROW; AVILA, 2000).

$\mathrm{O}$ aumento na procura de plantas medicinais frente à oferta insuficiente das mesmas conduziu a uma queda de sua qualidade. Muitos produtores desconhecem os cuidados que se deve ter nas diversas etapas para a obtenção de matérias-primas e/ou produtos de qualidade adequada e não contam com a orientação de profissionais capacitados.

A identificação e a pureza da droga, vegetal, assim como a avaliação de seus princípios ativos, são tarefas indispensáveis àqueles que buscam obter produtos de boa qualidade (OLIVEIRA; AKISUE; AKISUE, 1991). No Brasil, apesar do grande consumo de produtos derivados de plantas, os produtos comercializados e consumidos não estavam sujeitos a nenhum tipo de controle. Em 1995, o Ministério da Saúde instituiu a Portaria MS/SNVS no 6, de 31 de janeiro de 1995, (BRASIL, 1995) que regulamentava o registro de produtos fitoterápicos para fins comerciais; depois veio a RDC 17 (BRASIL, 2000) e, mais recentemente, a RDC 48 (BRASIL, 2004), que reafirma, definitivamente, que fitoterápicos são medicamentos e, desta forma, resgata a necessidade da existência de estudos de segurança, eficácia e qualidade, prévios ao registro desses produtos.

Segundo Araújo e Ohara (2000), investigações da qualidade microbiológica de drogas vegetais e produtos delas obtidos, realizadas em outros países, mostraram índices de contaminação microbiana em desacordo com normas internacionalmente aceitas para medicamentos (LENOBLE et al., 1980; CORTEZ et al., 1986; FAVET, 1992; KEDZIA, 1995; MARTINS et al., 2001; KNEIFEL et al., 2002). No Brasil, avaliações de fitoterápicos comercializados em farmácias apresentaram resultados semelhantes (FISCHER; SAITO, 1988; FISCHER et al., 1993; SANTOS et al., 1995).

Este trabalho foi realizado em parceria com a Secretaria do Estado da Agricultura e Abastecimento (Projeto Paraná) e teve como objetivo a busca da melhoria da qualidade das plantas medicinais produzidas no Estado do Paraná, através do monitoramento das regiões produtoras, mediante análises físico-químicas e microbiológicas. Este trabalho abordará a qualidade das plantas medicinais do ponto de vista microbiológico, devido à potencialidade de contaminação das mesmas, considerando sua origem natural (FISCHER et al., 1996).

\section{MATERIAL E MÉTODOS}

\section{Material vegetal}

O material de estudo deste trabalho foram 72 amostras de plantas medicinais de 27 diferentes espécies, obtidas de sete regiões do Estado do Paraná. O levantamento das regiões produtoras de plantas medicinais do Estado do Paraná foi realizado com orientação da EMATER - PR (Empresa Paranaense de Assistência Técnica Rural). As espécies de interesse, de cada região produtora de plantas medicinais, foram selecionadas em função do volume produzido e importância de tal produção para cada região.

O material vegetal, após coleta ou colheita e secagem, era acondicionado em embalagens de papel Kraft duplo e polietileno atóxico. A amostra retirada para análise foi enviada ao controle microbiológico, em sacos plásticos, contendo aproximadamente $100 \mathrm{~g}$, juntamente com o protocolo de encaminhamento, onde os produtores informavam a forma de cultivo, de coleta ou colheita e tipo de processamento sofrido pelo material vegetal (secagem, envase, armazenamento).

Rev. Bras. Farmacogn., V. 14, n. 1, jan.-jun. 2004. 


\section{Pré-tratamento do material a ser examinado}

Foram suspensos $10 \mathrm{~g}$ de cada amostra em $90 \mathrm{~mL}$ de solução tampão de cloreto de sódiopeptona, ajustando-se o pH quando necessário para 7,0. A amostra tratada correspondeu a diluição $10^{-1}$ (WORLD HEALTH ORGANIZATION, 1998).

\section{Determinação numérica de formas viáveis}

Contagem de bactérias aeróbicas: as amostras previamente tratadas foram diluídas em séries decimais, empregando a mesma solução utilizada no tratamento, até que fossem obtidas contagens inferiores a 300UFC (Unidades Formadoras de Colônias). Cada diluição foi plaqueada em duplicata, empregando-se a técnica oficial da semeadura em profundidade no ágar caseínasoja. A incubação foi feita a 30-35 $\mathrm{C}$, por 48h (WORLD HEALTH ORGANIZATION, 1998).

Contagem de bolores e leveduras: da mesma forma que para contagem de bactérias aeróbicas, foram preparadas diluições decimais das amostras previamente tratadas, visando obter uma contagem esperada de, no máximo, 100UFC. A técnica empregada foi a da semeadura em profundidade no ágar Sabouraud-dextrose, acrescido de uma solução de ácido tartárico 10\% até obtenção de $\mathrm{pH} 3,0-3,5$. Cada diluição foi plaqueada em duplicata e incubada a $20-25^{\circ} \mathrm{C}$, por 5 dias (WORLD HEALTH ORGANIZATION, 1998).

\section{Pesquisa de patógenos específicos}

A pesquisa de patógenos específicos: Enterobactérias e outras bactérias Gram negativas, (Escherichia coli, Salmonella sp., Pseudomonas aeruginosa e Staphylococcus aureus) consistiu na transferência de $10 \mathrm{ml}$ do material previamente tratado e incubado a $30-37^{\circ} \mathrm{C}$, por período variável entre 2 a 5 h, para um caldo de enriquecimento seletivo ou não seletivo, posterior subcultura em placa para isolamento e diferenciação, visando a identificação do microrganismo em questão, através de provas bioquímicas e sorológicas. O material previamente tratado não foi incubado no caso da pesquisa de Pseudomonas aeruginosa e Staphylococcus aureus, sendo diretamente transferido para caldo de enriquecimento não seletivo (WORLD HEALTH ORGANIZATION, 1998). O caldo de enriquecimento e o meio de cultura utilizados para subcultura, bem como o tempo e a temperatura de incubação destes, diferiram conforme o microrganismo a ser pesquisado, e estão relatados na Tabela 1. A identificação dos microrganismos acima citados, quando detectados, foi realizada conforme descrito pela Organização Mundial da Saúde (OMS) (WORLD HEALTH ORGANIZATION, 1998).

Tabela 1. Informações sobre as metodologias usadas para a pesquisa microbiológica

\begin{tabular}{l|l|l|l||l|}
\hline Patógenos específicos & \multicolumn{1}{|c|}{$\begin{array}{c}\text { Caldo de } \\
\text { enriquecimento }\end{array}$} & $\begin{array}{c}\text { Temperatura / } \\
\text { tempo de } \\
\text { incubação }\end{array}$ & $\begin{array}{c}\text { Meio de cultura } \\
\text { (subcultura) }\end{array}$ & $\begin{array}{c}\text { Temperatura / } \\
\text { tempo de } \\
\text { incubação }\end{array}$ \\
\hline \begin{tabular}{l|l|l|l|} 
Enterobactérias e outras \\
bactérias Gram negativas \\
(detecção de presença)
\end{tabular} & Mossel & $35-37^{\circ} \mathrm{C} / 18-48 \mathrm{~h}$ & Ágar VRB & $35-37^{\circ} \mathrm{C} / 18-48 \mathrm{~h}$ \\
\hline Escherichia coli & Mac Conkey & $43-45^{\circ} \mathrm{C} / 18-24 \mathrm{~h}$ & Ágar Mac Conkey & $43-45^{\circ} \mathrm{C} / 18-24 \mathrm{~h}$ \\
\hline Salmonella sp. & Tetrationato & $42-43^{\circ} \mathrm{C} / 18-24 \mathrm{~h}$ & Ágar Rambach & $35-37^{\circ} \mathrm{C} / 24-28 \mathrm{~h}$ \\
\hline Pseudomonas aeruginosa & Caseína-soja & $35-37^{\circ} \mathrm{C} / 24-48 \mathrm{~h}$ & Ágar Cetrimide & $35-37^{\circ} \mathrm{C} / 24-48 \mathrm{~h}$ \\
\hline Staphylococcus aureus & Caseína-soja & $35-37^{\circ} \mathrm{C} / 24-48 \mathrm{~h}$ & Ágar Baird-Parker & $35-37^{\circ} \mathrm{C} / 24-48 \mathrm{~h}$ \\
\hline
\end{tabular}




\section{RESULTADOS}

A Tabela 2 apresenta a listagem das plantas medicinais analisadas, acompanhadas de seus nomes científicos, lote, região produtora, e resultados da análise microbiológica, a qual compreende contagem de microrganismos aeróbios totais, contagem de bolores e leveduras, pesquisa de enterobactérias e outras bactérias Gram negativas.

Tabela 2. Resultados da análise microbiológica das plantas medicinais produzidas em diferentes regiões do Estado do Paraná, em 2002.

\begin{tabular}{|c|c|c|c|c|c|c|c|c|c|c|}
\hline Nome popular & $\begin{array}{c}\text { Nome } \\
\text { Científico }\end{array}$ & Lote & Região* & $\begin{array}{l}\text { Contagem } \\
\text { de aeróbios } \\
\text { totais UFC/g }\end{array}$ & $\begin{array}{l}\text { Contagem } \\
\text { de bolores } \\
\text { e leveduras } \\
\text { UFC/g }\end{array}$ & $\underset{* *}{\mathrm{PE}}$ & $\underset{* *}{P E C}$ & $\underset{* *}{\text { PSal }}$ & $\underset{* *}{\mathrm{PSau}}$ & $\underset{* \star}{\text { PPaeru }}$ \\
\hline 1. Macela & $\begin{array}{l}\text { Achyrocline } \\
\text { satureloides }\end{array}$ & - & Bom Jesus do Sul ${ }^{5}$ & $2,0 \times 10^{2}$ & $4,8 \times 10^{4}$ & $\mathrm{P}$ & $A$ & $A$ & $A$ & A \\
\hline 2. Menta & Mentha arvensis & - & Bom Jesus do Sul ${ }^{5}$ & $2,0 \times 10^{2}$ & $1,0 \times 10^{2}$ & $\mathrm{P}$ & A & $A$ & $A$ & A \\
\hline 3. Melissa & $\begin{array}{l}\text { Melissa } \\
\text { officinalis }\end{array}$ & - & Bom Jesus do Sul ${ }^{5}$ & $4,0 \times 10^{3}$ & $1,5 \times 10^{2}$ & $\mathrm{P}$ & A & $A$ & $A$ & $\mathrm{P}$ \\
\hline 4. Camomila & $\begin{array}{l}\text { Chamomilla } \\
\text { recutita }\end{array}$ & - & Bom Jesus do Sul ${ }^{5}$ & $3,1 \times 10^{4}$ & $1,0 \times 10^{4}$ & $\mathrm{P}$ & $\mathrm{P}$ & $A$ & A & A \\
\hline 5. Alecrim & $\begin{array}{l}\text { Rosmarinus } \\
\text { officinalis }\end{array}$ & $201 / 1$ & Campo Magro $^{4}$ & $1,0 \times 10^{3}$ & $4,5 \times 10^{3}$ & $\mathrm{P}$ & A & $A$ & $A$ & A \\
\hline 6. Tanchagem & Plantago sp & $275 / 3$ & Campo Magro $^{4}$ & $1,0 \times 10^{3}$ & $<10^{3}$ & A & A & $A$ & $A$ & A \\
\hline 7. Melissa & $\begin{array}{l}\text { Melissa } \\
\text { officinalis }\end{array}$ & $258 / 3$ & Campo Magro $^{4}$ & $6,3 \times 10^{5}$ & $4,6 \times 10^{5}$ & $\mathrm{P}$ & A & $A$ & $A$ & A \\
\hline 8. Melissa & $\begin{array}{l}\text { Melissa } \\
\text { officinalis }\end{array}$ & $260 / 1$ & Campo Magro $^{4}$ & $>10^{5}$ & $>10^{5}$ & $\mathrm{P}$ & A & $A$ & A & A \\
\hline 9. Melissa & $\begin{array}{l}\text { Melissa } \\
\text { officinalis }\end{array}$ & $261 / 1$ & Campo Magro $^{4}$ & $7,3 \times 10^{4}$ & $4,2 \times 10^{6}$ & $\mathrm{P}$ & $P$ & $A$ & A & $A$ \\
\hline 10. Camomila & $\begin{array}{l}\text { Chamomilla } \\
\text { recutita }\end{array}$ & 1 & Campo Magro $^{3}$ & $2,3 \times 10^{6}$ & $2,1 \times 10^{5}$ & $\mathrm{P}$ & A & A & $A$ & $A$ \\
\hline 11. Camomila & $\begin{array}{l}\text { Chamomilla } \\
\text { recutita }\end{array}$ & 2 & Campo Magro $^{2}$ & $1,8 \times 10^{6}$ & $4,0 \times 10^{5}$ & $\mathrm{P}$ & $P$ & $A$ & A & A \\
\hline 12. Camomila & $\begin{array}{l}\text { Chamomilla } \\
\text { recutita }\end{array}$ & 3 & Campo Magro $^{1}$ & $6,4 \times 10^{5}$ & $3,8 \times 10^{5}$ & $\mathrm{P}$ & $P$ & A & $A$ & $A$ \\
\hline 13. Camomila & $\begin{array}{l}\text { Chamomilla } \\
\text { recutita }\end{array}$ & 4 & $\begin{array}{l}\text { Campo Magro } \\
\text { Testemunha }\end{array}$ & $6,8 \times 10^{5}$ & $2,4 \times 10^{5}$ & $\mathrm{P}$ & $\mathrm{P}$ & $A$ & $A$ & $A$ \\
\hline 14. Camomila & $\begin{array}{l}\text { Chamomilla } \\
\text { recutita }\end{array}$ & 5 & Campo Magro $^{2}$ & $3,1 \times 10^{5}$ & $5,1 \times 10^{5}$ & $\mathrm{P}$ & A & $A$ & $A$ & A \\
\hline 15. Camomila & $\begin{array}{l}\text { Chamomilla } \\
\text { recutita }\end{array}$ & 6 & Campo Magro $^{1}$ & $3,0 \times 10^{5}$ & $3,1 \times 10^{5}$ & $\mathrm{P}$ & $P$ & $A$ & $A$ & A \\
\hline 16. Camomila & $\begin{array}{l}\text { Chamomilla } \\
\text { recutita }\end{array}$ & 7 & Campo Magro $^{3}$ & $2,8 \times 10^{6}$ & $5,4 \times 10^{5}$ & $\mathrm{P}$ & $A$ & A & $A$ & $A$ \\
\hline 17. Camomila & $\begin{array}{l}\text { Chamomilla } \\
\text { recutita }\end{array}$ & 8 & $\begin{array}{l}\text { Campo Magro } \\
\text { Testemunha }\end{array}$ & $7,3 \times 10^{5}$ & $6,5 \times 10^{5}$ & $\mathrm{P}$ & A & $A$ & $A$ & $A$ \\
\hline 18. Camomila & $\begin{array}{l}\text { Chamomilla } \\
\text { recutita }\end{array}$ & 9 & Campo Magro $^{1}$ & $1,6 \times 10^{6}$ & $2,6 \times 10^{5}$ & $\mathrm{P}$ & $\mathrm{P}$ & $A$ & $A$ & A \\
\hline 19. Camomila & $\begin{array}{l}\text { Chamomilla } \\
\text { recutita }\end{array}$ & 10 & $\begin{array}{l}\text { Campo Magro } \\
\text { Testemunha }\end{array}$ & $1,2 \times 10^{6}$ & $6,9 \times 10^{5}$ & $\mathrm{P}$ & $A$ & $A$ & $A$ & $A$ \\
\hline 20. Camomila & $\begin{array}{l}\text { Chamomilla } \\
\text { recutita }\end{array}$ & 11 & Campo Magro $^{2}$ & $2,7 \times 10^{6}$ & $7,2 \times 10^{5}$ & $\mathrm{P}$ & A & $A$ & $A$ & A \\
\hline 21. Camomila & $\begin{array}{l}\text { Chamomilla } \\
\text { recutita }\end{array}$ & 12 & Campo Magro $^{3}$ & $7,7 \times 10^{5}$ & $2,5 \times 10^{5}$ & $\mathrm{P}$ & $A$ & $A$ & $A$ & A \\
\hline 22. Camomila & $\begin{array}{l}\text { Chamomilla } \\
\text { recutita }\end{array}$ & 14 & Campo Magro $^{3}$ & $9,5 \times 10^{5}$ & $7,5 \times 10^{5}$ & $\mathrm{P}$ & A & $A$ & $A$ & A \\
\hline 23. Camomila & $\begin{array}{l}\text { Chamomilla } \\
\text { recutita }\end{array}$ & 15 & $\begin{array}{l}\text { Campo Magro } \\
\text { Testemunha }\end{array}$ & $3,2 \times 10^{5}$ & $2,5 \times 10^{5}$ & $\mathrm{P}$ & $A$ & $A$ & $A$ & $A$ \\
\hline
\end{tabular}

Rev. Bras. Farmacogn., V. 14, n. 1, jan.-jun. 2004. 


\begin{tabular}{|c|c|c|c|c|c|c|c|c|c|c|}
\hline 24. Camomila & $\begin{array}{l}\text { Chamomilla } \\
\text { recutita }\end{array}$ & 16 & Campo Magro $^{2}$ & $5,5 \times 10^{5}$ & $3,6 \times 10^{5}$ & $\mathrm{P}$ & $A$ & $A$ & $A$ & A \\
\hline 25. Camomila & $\begin{array}{l}\text { Chamomilla } \\
\text { recutita }\end{array}$ & A & $\begin{array}{l}\text { Campo Magro } \\
\text { Testemunha }\end{array}$ & $1,2 \times 10^{6}$ & $4,7 \times 10^{5}$ & $\mathrm{P}$ & $\mathrm{P}$ & $A$ & $A$ & A \\
\hline 26. Camomila & $\begin{array}{l}\text { Chamomilla } \\
\text { recutita }\end{array}$ & $\mathrm{B}$ & Campo Magro $^{1}$ & $5,2 \times 10^{5}$ & $5,1 \times 10^{5}$ & $\mathrm{P}$ & $\mathrm{P}$ & $A$ & $A$ & A \\
\hline 27. Camomila & $\begin{array}{l}\text { Chamomilla } \\
\text { recutita }\end{array}$ & C & Campo Magro $^{2}$ & $6,8 \times 10^{5}$ & $8,0 \times 10^{5}$ & $\mathrm{P}$ & $A$ & $A$ & $A$ & A \\
\hline 28. Camomila & $\begin{array}{l}\text { Chamomilla } \\
\text { recutita }\end{array}$ & $\mathrm{D}$ & Campo Magro $^{3}$ & $<10^{4}$ & $4,5 \times 10^{6}$ & $\mathrm{P}$ & $\mathrm{P}$ & $A$ & $A$ & A \\
\hline 29. Cavalinha & $\begin{array}{l}\text { Equisetum } \\
\text { arvense }\end{array}$ & CEN 05/01 & Central Regional $^{4}$ & $8,0 \times 10^{5}$ & $2,0 \times 10^{6}$ & $\mathrm{P}$ & A & $A$ & A & $\mathrm{P}$ \\
\hline 30. Camomila & $\begin{array}{l}\text { Chamomilla } \\
\text { recutita }\end{array}$ & $299 / 3$ & Curitiba $^{4}$ & $9,0 \times 10^{5}$ & $<10^{3}$ & $\mathrm{P}$ & $A$ & $A$ & $A$ & A \\
\hline 31. Camomila & $\begin{array}{l}\text { Chamomilla } \\
\text { recutita var. } \\
\text { Itália }\end{array}$ & 1B & Pinhais $\mathrm{PCB}^{1}$ & $1,8 \times 10^{4}$ & $1,3 \times 10^{4}$ & $\mathrm{P}$ & $\mathrm{P}$ & A & $A$ & A \\
\hline 32. Pacová & $\begin{array}{l}\text { Renealmia } \\
\text { exaltata }\end{array}$ & Único & Pinhais $^{1}$ & $2,4 \times 10^{5}$ & $1,2 \times 10^{5}$ & $\mathrm{P}$ & $A$ & $A$ & $A$ & A \\
\hline 33. Caité & Canna coccinea & - & Pinhais $^{1}$ & $2,3 \times 10^{6}$ & $1,6 \times 10^{5}$ & $\mathrm{P}$ & $A$ & $A$ & $A$ & $\mathrm{P}$ \\
\hline 34. Alfavaca & $\begin{array}{l}\text { Ocimum } \\
\text { basilicum }\end{array}$ & - & Pinhais $^{1}$ & $4,4 \times 10^{4}$ & $2,0 \times 10^{4}$ & $\mathrm{P}$ & A & $A$ & $A$ & $\mathrm{P}$ \\
\hline 35. Yacon & $\begin{array}{l}\text { Polyimnia } \\
\text { sonchifolia }\end{array}$ & - & Pinhais $^{1}$ & $3,1 \times 10^{5}$ & $3,2 \times 10^{4}$ & $\mathrm{P}$ & A & A & A & A \\
\hline 36. Fáfia & $\begin{array}{l}\text { Pfaffia } \\
\text { glomerata }\end{array}$ & $\begin{array}{c}\text { Raiz } \\
\text { fatiada }\end{array}$ & Pinhais $^{1}$ & $1,3 \times 10^{3}$ & $1,5 \times 10^{2}$ & $A$ & $A$ & $A$ & $A$ & A \\
\hline 37. Fáfia & \begin{tabular}{|l} 
Pfaffia \\
glomerata
\end{tabular} & Raiz ralada & Pinhais $^{1}$ & $4,6 \times 10^{6}$ & $3,7 \times 10^{6}$ & $\mathrm{P}$ & $A$ & $A$ & $A$ & A \\
\hline 38. Menta & Mentha piperita & $876 / 2$ & Prudentópolis ${ }^{4}$ & $9,3 \times 10^{5}$ & $1,1 \times 10^{5}$ & $\mathrm{P}$ & $A$ & A & $A$ & $\mathrm{P}$ \\
\hline 39. Maracujá & Passiflora edulis & $896 / 2$ & Prudentópolis ${ }^{4}$ & $4,0 \times 10^{3}$ & $1,0 \times 10^{3}$ & $\mathrm{P}$ & A & A & A & A \\
\hline 40. Maracujá & Passiflora edulis & $936 / 2$ & Prudentópolis ${ }^{4}$ & $2,3 \times 10^{6}$ & $4,2 \times 10^{6}$ & $\mathrm{P}$ & $A$ & $A$ & $A$ & A \\
\hline 41. Maracujá & Passiflora edulis & $913 / 2$ & Prudentópolis ${ }^{4}$ & $>10^{5}$ & $3,3 \times 10^{6}$ & $A$ & $A$ & $A$ & $A$ & $\mathrm{P}$ \\
\hline 42. Maracujá & Passiflora edulis & $911 / 2$ & Prudentópolis ${ }^{4}$ & $4,2 \times 10^{4}$ & $1,0 \times 10^{4}$ & $\mathrm{P}$ & A & A & A & A \\
\hline 43. Maracujá & Passiflora edulis & $922 / 2$ & Prudentópolis ${ }^{4}$ & $>10^{5}$ & $>10^{5}$ & $\mathrm{P}$ & $A$ & A & A & $\mathrm{P}$ \\
\hline 44. Macela & $\begin{array}{l}\text { Achyrocline } \\
\text { satureoides }\end{array}$ & $894 / 2$ & Prudentópolis ${ }^{4}$ & $1,0 \times 10^{3}$ & $1,0 \times 10^{5}$ & $\mathrm{P}$ & $A$ & A & $A$ & A \\
\hline 45. Alcachofra & $\begin{array}{l}\text { Cynara } \\
\text { scolymus }\end{array}$ & $779 / 2$ & Prudentópolis ${ }^{4}$ & $4,9 \times 10^{6}$ & $1,0 \times 10^{6}$ & $\mathrm{P}$ & $A$ & A & $A$ & $\mathrm{P}$ \\
\hline 46. Alcachofra & $\begin{array}{l}\text { Cynara } \\
\text { scolymus }\end{array}$ & $906 / 2$ & Prudentópolis ${ }^{4}$ & $9,9 \times 10^{6}$ & $3,0 \times 10^{6}$ & $\mathrm{P}$ & $A$ & $A$ & $A$ & $\mathrm{P}$ \\
\hline 47. Alcachofra & $\begin{array}{l}\text { Cynara } \\
\text { scolymus }\end{array}$ & $909 / 2$ & Prudentópolis ${ }^{4}$ & $1,4 \times 10^{6}$ & $2,2 \times 10^{6}$ & $\mathrm{P}$ & $A$ & A & A & A \\
\hline $\begin{array}{l}\text { 48. Capim } \\
\text { Limão }\end{array}$ & $\begin{array}{l}\text { Cymbopogon } \\
\text { citratus }\end{array}$ & $908 / 2$ & Prudentópolis ${ }^{4}$ & $1,7 \times 10^{7}$ & $>10^{5}$ & $\mathrm{P}$ & $A$ & A & $A$ & $\mathrm{P}$ \\
\hline $\begin{array}{l}\text { 49. Capim } \\
\text { Limão }\end{array}$ & $\begin{array}{l}\text { Cymbopogon } \\
\text { citratus }\end{array}$ & $916 / 2$ & Prudentópolis ${ }^{4}$ & $1,6 \times 10^{7}$ & $2,1 \times 10^{6}$ & $\mathrm{P}$ & $A$ & $A$ & $A$ & $\mathrm{P}$ \\
\hline $\begin{array}{l}\text { 50. Capim } \\
\text { Limão }\end{array}$ & $\begin{array}{l}\text { Cymbopogon } \\
\text { citratus }\end{array}$ & $898 / 2$ & Prudentópolis ${ }^{4}$ & $>10^{5}$ & $2,3 \times 10^{6}$ & $\mathrm{P}$ & $A$ & $A$ & $A$ & $\mathrm{P}$ \\
\hline $\begin{array}{l}\text { 51. Capim } \\
\text { Limão }\end{array}$ & $\begin{array}{l}\text { Cymbopogon } \\
\text { citratus }\end{array}$ & $858 / 2$ & Prudentópolis ${ }^{4}$ & $>10^{5}$ & $8,4 \times 10^{6}$ & $\mathrm{P}$ & $A$ & A & A & $\mathrm{P}$ \\
\hline 52. Carqueja & $\begin{array}{l}\text { Baccharis } \\
\text { genistelloides }\end{array}$ & $778 / 2$ & Prudentópolis ${ }^{4}$ & $9,5 \times 10^{4}$ & $1,5 \times 10^{4}$ & $P$ & A & A & A & $\mathrm{P}$ \\
\hline 53. Carqueja & $\begin{array}{l}\text { Baccharis } \\
\text { genistelloides }\end{array}$ & $709 / 2$ & Prudentópolis ${ }^{4}$ & $1,0 \times 10^{3}$ & $2,5 \times 10^{3}$ & $P$ & A & A & A & A \\
\hline 54. Losna & $\begin{array}{l}\text { Artemisia } \\
\text { absinthium }\end{array}$ & $809 / 2$ & Prudentópolis ${ }^{4}$ & $1,0 \times 10^{3}$ & $<10^{3}$ & $\mathrm{P}$ & A & A & $A$ & A \\
\hline 55. Melissa & \begin{tabular}{|l|} 
Melissa \\
officinalis
\end{tabular} & $912 / 2$ & Prudentópolis ${ }^{4}$ & $>10^{5}$ & $>10^{5}$ & $\mathrm{P}$ & $A$ & A & $A$ & A \\
\hline
\end{tabular}




\begin{tabular}{|c|c|c|c|c|c|c|c|c|c|c|}
\hline 56. Melissa & $\begin{array}{l}\text { Melissa } \\
\text { officinalis }\end{array}$ & $906 / 2$ & Prudentópolis ${ }^{4}$ & $1,5 \times 10^{4}$ & $1,0 \times 10^{4}$ & $\mathrm{P}$ & $A$ & A & A & A \\
\hline 57. Melissa & $\begin{array}{l}\text { Melissa } \\
\text { officinalis }\end{array}$ & $931 / 2$ & Prudentópolis ${ }^{4}$ & $7,0 \times 10^{3}$ & $1,0 \times 10^{4}$ & $P$ & A & A & $A$ & A \\
\hline 58. Melissa & $\begin{array}{l}\text { Melissa } \\
\text { officinalis }\end{array}$ & $921 / 2$ & Prudentópolis ${ }^{4}$ & $8,0 \times 10^{4}$ & $5,5 \times 10^{4}$ & $P$ & $A$ & $A$ & $A$ & $\mathrm{P}$ \\
\hline 59. Cavalinha & Equisetum sp. & $312 / 3$ & Prudentópolis ${ }^{4}$ & $1,0 \times 10^{6}$ & $5,0 \times 10^{5}$ & $P$ & A & A & A & A \\
\hline $\begin{array}{l}\text { 60. Espinheira } \\
\text { Santa }\end{array}$ & $\begin{array}{l}\text { Maytenus } \\
\text { ilicifolia }\end{array}$ & $904 / 2$ & Prudentópolis ${ }^{4}$ & $4,5 \times 10^{3}$ & $1,0 \times 10^{4}$ & $P$ & A & $A$ & $A$ & A \\
\hline 61. Alecrim & $\begin{array}{l}\text { Rosmarinus } \\
\text { officinalis }\end{array}$ & VRS $10 / 00$ & São João do Ivaí ${ }^{4}$ & $1,4 \times 10^{4}$ & $7,5 \times 10^{3}$ & $P$ & $\mathrm{P}$ & $A$ & $A$ & A \\
\hline $\begin{array}{l}\text { 62. Capim } \\
\text { Cidreira }\end{array}$ & $\begin{array}{l}\text { Cymbopogon } \\
\text { citratus }\end{array}$ & VRS 09/01 & São João do Ivaí ${ }^{3}$ & $8,5 \times 10^{5}$ & $5,5 \times 10^{5}$ & $P$ & A & $A$ & A & A \\
\hline $\begin{array}{l}\text { 63. Espinheira } \\
\text { Santa }\end{array}$ & $\begin{array}{l}\text { Maytenus } \\
\text { aquifolium }\end{array}$ & VRS 08/01 & São João do Ivaí ${ }^{4}$ & $6,0 \times 10^{3}$ & $4,5 \times 10^{3}$ & $\mathrm{P}$ & $P$ & $A$ & A & A \\
\hline 64. Hortelã & Mentha arvensis & VRS 08/01 & São João do Ivaí ${ }^{3}$ & $4,3 \times 10^{6}$ & $2,0 \times 10^{5}$ & $\mathrm{P}$ & A & A & A & $\mathrm{P}$ \\
\hline 65. Porangaba & Cordia salicifolia & VRS $08 / 01$ & São João do Ivaí ${ }^{4}$ & $6,6 \times 10^{3}$ & $3,5 \times 10^{3}$ & $\mathrm{P}$ & $\mathrm{P}$ & $A$ & A & A \\
\hline $\begin{array}{l}\text { 66. Rosa } \\
\text { Branca }\end{array}$ & Rosa sp & VRS 01/01 & São João do Ivaí ${ }^{4}$ & $7,3 \times 10^{4}$ & $1,8 \times 10^{4}$ & $P$ & $P$ & $A$ & $A$ & A \\
\hline 67. Carqueja & $\begin{array}{l}\text { Baccharis } \\
\text { genistelloides }\end{array}$ & SBN 07/01 & Sítio Barra Nova ${ }^{3}$ & $<10^{3}$ & $2,6 \times 10^{4}$ & $\mathrm{P}$ & A & A & A & A \\
\hline 68. Camomila & $\begin{array}{l}\text { Matricaria } \\
\text { recutita }\end{array}$ & SBN 08/01 & Sítio Barra Nova ${ }^{3}$ & $1,2 \times 10^{4}$ & $2,8 \times 10^{3}$ & $\mathrm{P}$ & A & $A$ & $A$ & A \\
\hline 69. Centella & Centella asiatica & SBN 06/01 & Sítio Barra Nova ${ }^{3}$ & $6,5 \times 10^{3}$ & $5,0 \times 10^{3}$ & $P$ & A & A & $A$ & A \\
\hline 70. Ginseng & $\begin{array}{l}\text { Pfaffia } \\
\text { paniculata }\end{array}$ & SBN 07/00 & Sítio Barra Nova ${ }^{3}$ & $8,0 \times 10^{3}$ & $3,0 \times 10^{3}$ & $\mathrm{P}$ & $P$ & $A$ & A & A \\
\hline 71. Manjerona & $\begin{array}{l}\text { Origanum } \\
\text { majorana }\end{array}$ & SBN 04/01 & Sítio Barra Nova ${ }^{3}$ & $4,0 \times 10^{3}$ & $5,0 \times 10^{3}$ & $\mathrm{P}$ & $A$ & $A$ & $A$ & A \\
\hline 72. Sálvia & Salvia officinalis & SBN 06/01 & Sítio Barra Nova ${ }^{3}$ & $2,0 \times 10^{3}$ & $3,8 \times 10^{3}$ & $\mathrm{P}$ & $A$ & $A$ & $A$ & $P$ \\
\hline
\end{tabular}

* Os números ao lado da região indicam o tipo de cultivo empregado (adubação): (1) Adubação verde; (2) Adubação verde + produto químico; (3) Produto químico; (4) Não foi relatado nada quanto ao cultivo; (5) Não foi utilizado produto químico; ** PE = Pesquisa de Enterobactérias e outras bactérias Gram negativas; PEc = Pesquisa de Escherichia coli; PSal = Pesquisa de Salmonella sp.; PSau = Pesquisa de Staphylococcus aureus; PPaeru = Pesquisa de Pseudomonas aeruginosa; $\mathrm{P}=$ presença; $\mathrm{A}$ = ausência.

Os resultados da análise microbiológica das plantas medicinais contidos na Tabela 2, juntamente com aqueles contidos na Tabela 3, que evidenciam os motivos pelos quais os vegetais não enquadraram-se nas especificações da OMS, possibilitaram a classificação numérica do enquadramento das plantas em cada uma das especificações da OMS, conforme a aplicação a que se destinam. Muitas delas encontram-se impróprias ao consumo humano, a não ser que sejam submetidas a um processo de descontaminação (Tabela 4).

Para melhor visualização do tipo de cultivo empregado no desenvolvimento das plantas medicinais, produzidas nas diversas regiões do Estado do Paraná, de forma a permitir a correlação de como o vegetal foi obtido e a contaminação nele presente, foi elaborada a Tabela 5. 
Tabela 3. Principais causas de reprovação das drogas vegetais, de acordo com as especificações da OMS.

\begin{tabular}{|c|c|}
\hline Motivos & $\begin{array}{l}\text { Quantidade de drogas vegetais fora } \\
\text { do limite especificado (\% do total) }\end{array}$ \\
\hline Contagens elevadas de aeróbios, bolores e leveduras & $23(34 \%)$ \\
\hline $\begin{array}{l}\text { Contagens elevadas de aeróbios, bolores e leveduras + } \\
\text { Pseudomonas aeruginosa }\end{array}$ & $11(16,5 \%)$ \\
\hline Contagens elevadas de bolores e leveduras & $10(15 \%)$ \\
\hline Contagens elevadas de bolores e leveduras + Escherichia coli & $8(12 \%)$ \\
\hline $\begin{array}{l}\text { Contagens elevadas de aeróbios, bolores e leveduras + } \\
\text { Escherichia coli }\end{array}$ & $7(10,5 \%)$ \\
\hline $\begin{array}{l}\text { Contagens elevadas de bolores e leveduras }+ \text { Pseudomonas } \\
\text { aeruginosa }\end{array}$ & $4(6 \%)$ \\
\hline Contagens elevadas de aeróbios & $1(1,5 \%)$ \\
\hline Presença de Pseudomonas aeruginosa & $1(1,5 \%)$ \\
\hline Presença de Escherichia coli & $1(1,5 \%)$ \\
\hline $\begin{array}{l}\text { Contagens elevadas de aeróbios, bol. e leveduras + Pseudomonas } \\
\text { aeruginosa + Escherichia coli }\end{array}$ & $1(1,5 \%)$ \\
\hline
\end{tabular}

Tabela 4. Quantidade de plantas medicinais que enquadram-se nas especificações da OMS, conforme a aplicação.

\begin{tabular}{|c|c|c|c|}
\hline Limites da OMS & $\begin{array}{c}\text { Enquadram-se para } \\
\text { chá e uso interno }\end{array}$ & $\begin{array}{c}\text { Não enquadram-se para } \\
\text { chá e nem para uso } \\
\text { interno }\end{array}$ & $\begin{array}{c}\text { Enquadra-se para chá, mas } \\
\text { não para uso interno }\end{array}$ \\
\hline \begin{tabular}{c|c|c|} 
Quantidade de \\
plantas
\end{tabular} & $5(7 \%)$ & $57(79 \%)$ & $10(14 \%)$ \\
\hline
\end{tabular}

Tabela 5. Relação das regiões produtoras de plantas medicinais no Estado do Paraná e tipo de cultivo empregado.

\begin{tabular}{|l|c|c|c|c|c|c|}
\hline \begin{tabular}{l|l|l|l|} 
Regiões/Tipo de \\
cultivo
\end{tabular} & $\begin{array}{c}\text { Adubação } \\
\text { verde }\end{array}$ & $\begin{array}{c}\text { Adubação } \\
\text { verde }+ \\
\text { produto } \\
\text { químico }\end{array}$ & $\begin{array}{c}\text { Adubação } \\
\text { química }\end{array}$ & $\begin{array}{c}\text { Não foi } \\
\text { utilizado } \\
\text { produto } \\
\text { químico }\end{array}$ & $\begin{array}{c}\text { Não utiliza } \\
\text { nenhum } \\
\text { tipo de } \\
\text { produto }\end{array}$ & $\begin{array}{c}\text { Não foi relatado } \\
\text { nada quanto ao } \\
\text { cultivo }\end{array}$ \\
\hline Bom Jesus do Sul & - & - & - & $4(6 \%)$ & - & - \\
\hline Campo Magro & $4(6 \%)$ & $5(7 \%)$ & $5(7 \%)$ & - & $5(7 \%)$ & $5(7 \%)$ \\
\hline Central Regional & - & - & - & - & - & $1(1 \%)$ \\
\hline Curitiba & - & - & - & - & - & $1(1 \%)$ \\
\hline Pinhais - PCB & $4(6 \%)$ & - & - & $3(4 \%)$ & - & - \\
\hline Prudentópolis & - & - & - & - & - & $23(32 \%)$ \\
\hline São João do Ivaí & - & - & $2(3 \%)$ & - & - & $4(6 \%)$ \\
\hline Sítio Barra Nova & - & $2(3 \%)$ & $4(6 \%)$ & - & - & - \\
\hline Total & $8(11 \%)$ & $7(10 \%)$ & $11(15 \%)$ & $7(10 \%)$ & $5(7 \%)$ & $34(47 \%)$ \\
\hline
\end{tabular}




\section{DISCUSSÃO}

Os níveis encontrados de contaminação por microrganismos aeróbios totais variaram de $2,0 \times 10^{2} \mathrm{UFC} / \mathrm{g}$ a $1,7 \times 10^{7} \mathrm{UFC} / \mathrm{g}$, sendo que $45,83 \%$ das amostras apresentaram cargas entre $10^{5}$

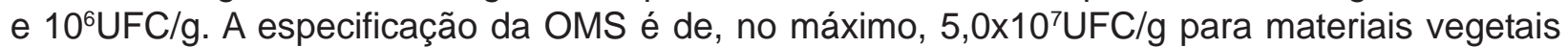

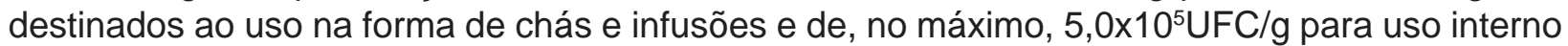
(WORLD HEALTH ORGANIZATION, 1998).

A diferença no grau de contaminação pode ser explicada pela distância da superfície do solo em que a planta cresce, isto é, plantas que crescem próximas ao solo apresentam maior carga microbiana. Além disto, certas plantas contêm barreiras naturais e substâncias antimicrobianas, que exercem efeito no crescimento microbiano (KNEIFEL et al., 2002).

A contaminação por bolores e leveduras das drogas vegetais analisadas variou de $1,0 \times 10^{2}$ a $8,4 \times 10^{6} \mathrm{UFC} / \mathrm{g}$, com maior freqüência de amostras com cargas de $10^{5} \mathrm{UFC} / \mathrm{g}(36,11 \%)$. A

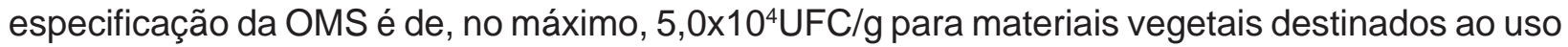

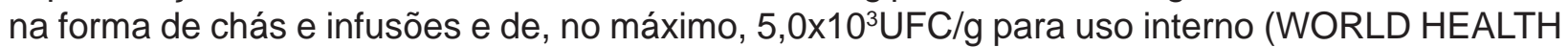
ORGANIZATION, 1998).

Contagens elevadas de fungos constituem um risco, em virtude da possibilidade desses serem produtores de micotoxinas, como a aflatoxina, que é uma substância cancerígena (ARAÚJO; OHARA, 2000). Hitokoto et al. (1978) demonstraram que fungos dos gêneros Penicillium, Aspergillus, Rhizopus, Mucor, Cladosporium e Aureobasidium spp. encontram-se freqüentemente associados à drogas vegetais; no entanto, fungos produtores de micotoxinas somente foram encontrados em um nível de $2 \%$. No entanto, Kumar e Roy (1993) detectaram níveis consideráveis de aflatoxinas, em várias amostras de plantas medicinais (KNEIFEL; CZECH; KOPP, 2002).

Foi detectada a presença de enterobactérias em quase todas as amostras (95,83\%). Em $22,22 \%$ das amostras analisadas, a presença de enterobactérias estava associada à presença de Escherichia coli, um coliforme fecal. Outros bacilos Gram-negativos encontrados foram: $E$. agglomerans, E. cloacae, E. aerogenes, E. gergoviae, C. amalonaticus, K. rhino e A. hinshawii. Segundo Araújo e Ohara (2000), Klebsiella sp., Enterobacter sp. e Citrobacter sp. são comuns em matérias-primas vegetais, uma vez que estão relacionados ao meio ambiente das plantas, não sendo necessariamente de origem fecal.

Em dezessete amostras (23,61\%), ocorreu crescimento de Pseudomonas aeruginosa, microrganismo que deve estar ausente, de acordo com as especificações da OMS. Este gênero faz parte da microflora do solo, mas pode, também, ser proveniente da microbiota das fossas nasais do responsável pelo plantio (TRABULSI et al., 1999; CETESB, 2002).

Salmonella sp. e Staphylococcus aureus não foram detectados nas amostras analisadas. Este último microrganismo não é comum neste tipo de material; apesar de produzir enterotoxinas em determinadas condições ambientais, ele não representa grande risco por via oral, desde que não esteja presente em grandes quantidades. Este tipo de matéria-prima parece não oferecer condições favoráveis para a sua multiplicação, uma vez que não se verificou na literatura qualquer relato de caso de intoxicação, decorrente da utilização de medicamentos contaminados com este microrganismo (ARAÚJO; OHARA, 2000).

Dentre as características microbiológicas que fizeram com que as plantas medicinais apresentassem contaminações, que ultrapassaram os limites microbianos estabelecidos pela Organização Mundial da Saúde, pôde-se evidenciar a contagem de aeróbios associada à contagem elevada de bolores e leveduras, que ocorreu em vinte e três amostras analisadas.

Das setenta e duas plantas analisadas, apenas cinco (7\%) enquadraram-se nos limites para materiais vegetais destinados ao uso na forma de chás e infusões ou para uso tópico e, ao mesmo tempo, nos limites para materiais de uso interno. Dez (14\%) enquadraram-se nos limites 
para materiais destinados ao uso na forma de chás e infusões ou para uso tópico, mas não encontraram-se dentro dos limites para uso interno. A maioria dos materiais, ou seja cinqüenta e sete deles (79\%), não se enquadrou em nenhum dos dois limites capazes de garantir a utilização de um material com a qualidade mínima necessária ao consumo humano.

As plantas medicinais do Estado do Paraná podem ser oriundas de cultivo tradicional, policultivo orgânico ou coleta da espécie nativa. Com cultivo tradicional, usando insumos agrícolas (adubo químico e agrotóxicos), destacam-se as regiões de Campo Magro, São João do Ivaí e Sítio Barra Nova. Já com cultivo orgânico, caracterizado pela ausência de insumos químicos, destacamse Campo Magro e Pinhais.

Nos protocolos encaminhados com as informações da forma de cultivo (53\% do total), foi possível observar que das oito plantas cultivadas em solo adubado organicamente, seis apresentaram contagens de microrganismos aeróbios, bolores e leveduras acima do especificado. Destas seis, cinco, também apresentaram crescimento de Escherichia coli, um coliforme fecal, provavelmente proveniente do preparo inadequado do adubo orgânico. Este microrganismo pode, ainda, ser oriundo de solo contaminado ou de água contaminada utilizada para irrigação, uma vez que mesmo nas plantas sem adubação, ocorreu tal crescimento. As outras duas plantas apresentaram contaminação por Pseudomonas aeruginosa, além de contagens superiores ao limite máximo estabelecido pela OMS.

Das onze plantas desenvolvidas em solo adubado quimicamente, todas apresentaram contagem elevada de bolores e leveduras; cinco delas apresentaram ainda um grande número de aeróbios; uma apresentou contagem elevada de aeróbios e desenvolvimento de Pseudomonas aeruginosa; uma apresentou presença de Escherichia coli e outra de Pseudomonas aeruginosa.

Ante o exposto foi possível observar que as plantas oriundas de cultivo tradicional apresentaram menor porcentagem de contaminação por microrganismos aeróbios, Pseudomonas aeruginosa e Escherichia coli, sendo que a baixa contaminação por este último microrganismo evidencia que o mesmo deve ser proveniente da adubação orgânica.

Tais resultados permitiram afirmar que o cultivo orgânico, apesar de não deixar resíduos químicos prejudiciais à saúde, propiciou um maior nível de contaminação microbiológica do que o cultivo tradicional, destacando-se o fato do cultivo tradicional ser realizado em maior número de regiões que o cultivo orgânico.

Alguns cuidados nas etapas subseqüentes do processamento de plantas medicinais são necessários a fim de reduzir a carga microbiana: higiene adequada das mãos dos manipuladores das plantas medicinais, o material coletado deve ser colocado sobre uma superfície limpa, o recipiente de coleta deve ser limpo, deve-se procurar eliminar impurezas que possam acompanhar o órgão ou a planta recém-coletada, a secagem da planta deve ser realizada o mais rápido possível sem, entretanto, deixar de ser eficiente, dificultando a contaminação microbiológica, que é provavelmente favorecida, uma vez que a matéria-prima é novamente manipulada após a colheita. O local de secagem deve ser limpo, bem ventilado, protegido do ataque de insetos e de outros animais e, ainda, ao abrigo da luz. Após, o material deve ser acondicionado em embalagens adequadamente limpas, não esmagado e armazenado em lugar seco, ventilado, e protegido da incidência de raios solares e da entrada de roedores e insetos. Não deve haver o contato direto da embalagem com o chão, e esta deve ser colocada sobre um pallet, para evitar transferência de umidade e contato com possíveis animais (OLIVEIRA et al., 1991; REIS; MARIOT, 2001).

Os resultados observados neste estudo mostraram que a baixa qualidade microbiológica das drogas vegetais encontradas no comércio, nem sempre deve-se a descuidos na comercialização, e a elevada contaminação pode ser proveniente das inúmeras etapas da produção.

O treinamento dos agricultores por profissionais capacitados faz-se necessário, visto tratarse de matéria-prima utilizada para a produção de medicamentos. Fatos observados na prática, 
como a presença de fragmentos de insetos, terra, madeira, e de contaminantes microbiológicos, indicam que deve-se tomar medidas educativas urgentes, que contemplem uma abordagem multiprofissional de tal treinamento, comungando na busca da qualidade, em prol da eficácia e segurança terapêutica.

\section{REFERÊNCIAS}

ARAÚJO, A. da L. A. de; OHARA, M. T. Qualidade microbiológica de drogas vegetais comercializadas em feiras de São Paulo e de infusos derivados. Revista Brasileira de Ciências Farmacêuticas, v.36, n.1, p.129$137,2000$.

BRASIL, Ministério da Saúde, Secretaria Nacional de Vigilância Sanitária. Portaria no 6. Diário Oficial da Repúplica Federativa do Brasil, 31 jan. 1995. Institui e normaliza o registro de produtos fitoterápicos

BRASIL, Ministério da Saúde, Agência Nacional de Vigilância Sanitária. RDC no 17. Diário Oficial da República Federativa do Brasil, 24 abr. 2000. Aprova regulamento técnico, normatizando o registro de medicamentos fitoterápicos junto ao Sistema de Vigilância Sanitária.

BRASIL, Ministério da Saúde, Agência Nacional de Vigilância Sanitária. RDC no 48. Diário Oficial da República Federativa do Brasil, 18 mar. 2004. Dispõe sobre o registro dos medicamentos fitoterápicos.

CETESB. Microbiologia Ambiental. São Paulo: CETESB, 2002.

CORTEZ, Y.; PERSONNÉ, J. C.; ESTÈVE, G. Étude comparative de la contamination des plantes médicinales. Plantes Médicinales et Phytotherapie, v.20, n.3, p.236-250, 1986 apud ARAÚJO, A. da L. A. de; OHARA, M. T. Qualidade microbiológica de drogas vegetais comercializadas em feiras de São Paulo e de infusos derivados. Revista Brasileira de Ciências Farmacêuticas, v.36, n.1, p.129-137, 2000.

FAVET, J. Étude de la contamination microbienne d'une vingtaine de drogues végétales. Pharmacia Acta Helvetica, v.67, n.9-10, p.250-258, 1992 apud ARAÚJO, A. da L. A. de; OHARA, M. T. Qualidade microbiológica de drogas vegetais comercializadas em feiras de São Paulo e de infusos derivados. Revista Brasileira de Ciências Farmacêuticas, v.36, n.1, p.129-137, 2000.

FETROW, C. W.; AVILA, J. R. Manual de medicina alternativa para o profissional. Rio de Janeiro: Guanabara Koogan, 2000.

FISCHER, D. C. H.; SAITO, T. Contaminação microbiana de fitoterápicos. Revista de Farmácia e Bioquímica da Universidade de São Paulo, v.24, n.2, p.143-144, 1988.

FISCHER, D. C. H.; OHARA, M. T.; SAITO, T. Contaminação microbiana de medicamentos fitoterápicos sob a forma sólida. Revista de Farmácia e Bioquímica da Universidade de São Paulo, v.29, n.2, p.81-88, 1993

FISCHER, D. C. H.; OHARA, M. T.; SAITO, T. Padrão microbiano de medicamentos não estéreis de uso oral. Revista Brasileira de Farmacognosia, v.6, n.1, p.29-54, 1996.

HITOKOTO, H.; MOROZUMI, S.; WAUKE, T.; SAKAI, S.; KURATA, H. Fungal contamination and mycotoxin detection of powdered herbal drugs. Applied and Environmental Microbiology, v.36, p.252-256, 1978 apud KNEIFEL, W.; CZECH, E.; KOPP, B. Microbial contamination of medicinal plants. A review. Planta Medica, v.68, p.5-15, 2002.

KEDZIA, B. Microbiological purity of infusion in the light of the obligatory requirements. Herbal Polonia, v.41, n.4, p.190-197, 1995 apud ARAÚJO, A. da L. A. de; OHARA, M. T. Qualidade microbiológica de drogas vegetais comercializadas em feiras de São Paulo e de infusos derivados. Revista Brasileira de Ciências Farmacêuticas, v.36, n.1, p.129-137, 2000.

KNEIFEL, W.; CZECH, E.; KOPP, B. Microbial contamination of medicinal plants. A review. Planta Medica, v68, p.5-15, 2002.

KUMAR, S.; ROY, A. K. Ocurrence of aflatoxin in some liver curative herbal medicines. Letters in Applied Microbiology, v.17, p.112-114, 1993 apud KNEIFEL, W.; CZECH, E.; KOPP, B. Microbial contamination of medicinal plants. A review. Planta Medica, v.68, p.5-15, 2002.

Rev. Bras. Farmacogn., V. 14, n. 1, jan.-jun. 2004. 
LENOBLE, M.; FOURNIAT, J.; BOURLIOUX, P.; PARIS, M.; MAGHAMI, P.; GERMAN, A. Contrôle de la qualité microbiologique d'échatillons de Mentha piperita de diverses origines géographiques. Annales Pharmaceutiques Françaises, v.38, n.4, p.333-342, 1980.

LUZ, S. F. B.; SATO, M. E. O.; DUARTE, M. do R.; SANTOS, C. A. de M. Parâmetros para o controle de qualidade de folhas de Casearia sylvestris, guaçatonga. Revista Brasileira de Farmacognosia, v.7/8, n.1/2, p.1-11, 1997/1998.

MARTINS, H. M.; MARTINS, M. L.; DIAS, M. I.; BERNARDO, F. Evaluation of microbiological quality of medicinal plants used in natural infusions. International Journal of Food Microbiology, v.68, p.149-153, 2001.

OLIVEIRA, F. de; AKISUE, G.; AKISUE, M. K. Farmacognosia. São Paulo: Atheneu, 1991.

PINTO, T. de J. A.; KANEKO, T. M.; OHARA, M. T. Controle microbiológico de qualidade de produtos farmacêuticos, correlatos e cosméticos. São Paulo: Atheneu, 2000.

REIS, M. S. dos; MARIOT, A. Diversidade natural e aspectos agronômicos de plantas medicinais. In: SIMÕES, C. M. O.; SCHENKEL, E.P.; GOSMANN, G.; MELLO, J.C.P. DE; MENTZ, L.A.; PETROVICK, P.R. (org.) Farmacognosia: da planta ao medicamento. 3.ed. Porto Alegre: Editora da Universidade UFRGS; Florianópolis: Editora da UFSC, p. 41-62, 2001.

SANTOS, P. R. V. dos; OLIVEIRA, A. C. X. de; TOMASSINI, T. C. B. Controle microbiológico de produtos fitoterápicos. Revista de Farmácia e Bioquímica da Universidade de São Paulo, v.31, n.1, p.35-38, 1995.

TRABULSI, L. R.; ALTERTHUM, F.; GOMPERTZ, O. F.; CANDEIAS, J. A. N. Microbiologia. 3.ed. São Paulo: Atheneu, 1999.

WORLD HEALTH ORGANIZATION. Quality control methods for medicinal plant materials. Geneva: WHO, 1998.

${ }^{*}$ Autor para correspondência:

Profa. Dra. Mariella Zaroni

Laboratório de Controle de Qualidade

Departamento de Farmácia

Universidade Federal do Paraná

Av. Pref. Lothário Meissner, 3400

Curitiba - PR, CEP 80210-170.

E-mail: marizaroni@bol.com.br 